\title{
On the Existence of Directional Derivatives for Strongly Cone-Paraconvex Mappings
}

\author{
Ewa Bednarczuk ${ }^{1,2} \cdot$ Krzysztof Leśniewski $^{1,2}$ (D)
}

Received: 23 August 2017 / Accepted: 11 February 2018 / Published online: 20 March 2018

(C) The Author(s) 2018

\begin{abstract}
We investigate the existence of directional derivatives for strongly coneparaconvex mappings. Our result is an extension of the classical Valadier result on the existence of the directional derivative for cone convex mappings with values in weakly sequentially complete Banach spaces.
\end{abstract}

Keywords Directional derivative $\cdot$ Normal cones $\cdot$ Strongly paraconvex mappings $\cdot$ Cone convex mappings - Weakly sequentially complete Banach spaces

Mathematics Subject Classification (2010) Primary 49J50 · Secondary 52A41

\section{Introduction}

The concepts of approximate convexity for extended real-valued functions include among others, $\gamma$-paraconvexity [3, 4, 7, 8], $\gamma$-semiconcavity [1], $\alpha$-paraconvexity, strong $\alpha$ paraconvexity [9], semiconcavity [1], and approximate convexity [6]. Relations between these concepts were investigated by Rolewicz [7-9], Daniilidis and Georgiev [2], and Tabor

Dedicated to Michel Théra on the occasion of his 70th birthday.

Krzysztof Leśniewski

k.lesniewski@mini.pw.edu.pl

Ewa Bednarczuk

Ewa.Bednarczuk@ibspan.waw.pl; E.Bednarczuk@mini.pw.edu.pl

1 Systems Research Institute PAS, ul. Newelska 6, 01-447 Warszawa, Poland

2 Faculty of Mathematics and Information Science, Warsaw University of Technology, ul. Koszykowa 75, 00-662 Warsaw, Poland 
and Tabor [11]. These concepts were used, e.g., in [1], to investigate Hamilton-Jacobi equation. In a series of papers [7-9], Rolewicz investigated Gâteaux and Fréchet differentiability of strongly $\alpha$-paraconvex, generalizing in this way the Mazur theorem (1933).

Generalization of the above concepts to vector-valued mappings with values in a general vector space $Y$ was given by Veselý and Zajicek [13-16], Valadier [12], and Rolewicz [10]. In the paper [10], Rolewicz defined vector-valued strongly $\alpha-k$ paraconvex mappings and investigated their Gateaux and Fréchet differentiability, where $k \in K$ and $K$ is a closed convex cone in a normed vector space $Y$.

Let $\alpha: \mathbb{R}_{+} \rightarrow \mathbb{R}_{+}$be a nondecreasing function satisfying the condition

$$
\lim _{t \rightarrow 0^{+}} \frac{\alpha(t)}{t}=0 \text {. }
$$

Let $X$ be a normed space and let $k \in K$. The mapping $F: X \rightarrow Y$ is strongly $\alpha-k$ paraconvex on a convex subset $A$ of $X$ if there exists a constant $C>0$ such that for every $x_{1}, x_{2} \in A$ and every $\lambda \in[0,1]$

$$
F\left(\lambda x_{1}+(1-\lambda) x_{2}\right) \leq_{K} \lambda F\left(x_{1}\right)+(1-\lambda) F\left(x_{2}\right)+C \min \{\lambda, 1-\lambda\} \alpha\left(\left\|x_{1}-x_{2}\right\|\right) k,
$$

where $x \leq_{K} y \Longleftrightarrow y-x \in K$. In the sequel, we use the notation $\leq$ if the cone $K$ is clear from the context.

The mapping $F: X \rightarrow Y$ is strongly $\alpha-K$ paraconvex on a convex subset $A$ of $X$ if for every $k \in K$ there exists a constant $C>0$ such that for every $x_{1}, x_{2} \in A$ and every $\lambda \in[0,1]$

$$
F\left(\lambda x_{1}+(1-\lambda) x_{2}\right) \leq_{K} \lambda F\left(x_{1}\right)+(1-\lambda) F\left(x_{2}\right)+C \min \{\lambda, 1-\lambda\} \alpha\left(\left\|x_{1}-x_{2}\right\|\right) k .
$$

A strongly $\alpha(\cdot)-K$ paraconvex mapping $F$ is called strongly cone-paraconvex if the cone $K$ and the function $\alpha$ are clear from the context. Since for every $\lambda \in[0,1]$

$$
\lambda(1-\lambda) \leq \min \{\lambda, 1-\lambda\} \leq 2 \lambda(1-\lambda)
$$

condition (1) can be equivalently rewritten as

$$
F\left(\lambda x_{1}+(1-\lambda) x_{2}\right) \leq_{K} \lambda F\left(x_{1}\right)+(1-\lambda) F\left(x_{2}\right)+2 C \lambda(1-\lambda) \alpha\left(\left\|x_{1}-x_{2}\right\|\right) k .
$$

Strong cone-paraconvexity generalizes the cone convexity. The mapping $F: X \rightarrow Y$ is $K$-convex on a convex subset $A$ of $X$ if for every $x_{1}, x_{2} \in A$ and every $\lambda \in[0,1]$

$$
F\left(\lambda x_{1}+(1-\lambda) x_{2}\right) \leq_{K} \lambda F\left(x_{1}\right)+(1-\lambda) F\left(x_{2}\right) .
$$

In the present paper, we investigate the existence of directional derivatives for strongly cone-paraconvex mappings. Our main result (Theorem 2) is a generalization of the theorem of Valadier [12] concerning directional differentiability of cone convex mappings.

\section{Preliminary Facts}

Let $Y^{*}$ be the dual space of $Y$ and $K^{*} \subset Y^{*}$ be the positive dual cone to $K$,

$$
K^{*}:=\left\{y^{*} \in Y^{*} \mid y^{*}(y) \geq 0 \forall y \in K\right\} .
$$

Clearly, if $F$ is a strongly $\alpha(\cdot)-k$ paraconvex mapping with constant $C>0$, then for every $y^{*} \in K^{*}$, the function $y^{*} \circ F$ is a strongly $\alpha(\cdot)$-paraconvex function with the constant $C \cdot y^{*}(k)$.

In a normed space $Y$, a cone $K$ is normal (see [12]) if there is a number $C>0$ such that

$$
0 \leq_{K} x \leq_{K} y \Rightarrow\|x\| \leq C\|y\| \quad \text { for all } x, y \in Y .
$$


Every normal cone is pointed, i.e., $K \cap(-K)=\{0\}$.

In [13], Veselỳ and Zajiček introduced the concept of d.c. (delta-convex) mappings acting between Banach spaces $X$ and $Y$. A mapping $F: X \rightarrow Y$ is d.c. if there exists a continuous convex function $g: X \rightarrow \mathbb{R}$ such that for every $y^{*} \in Y^{*}$ the function $y^{*} \circ F+g$ is a d.c. function, i.e., it is representable as a difference of two convex functions.

According to [15], $F$ is order d.c. if $F$ is representable as a difference of two cone convex mappings on $A$. Consequently, if the cone $K$ is normal, then $F$ is also weakly order d.c.

Moreover, if the range space $Y$ of an order d.c. mapping $F$ is ordered by a well-based cone $K$ (and this is true for $L_{1}(\mu)$ ), it is easy to show (see Proposition 4.1 [15]) that the mapping is then d.c. d.c.

In the example below, we show that any strongly $\|\cdot\|^{2}-k_{0}$-paraconvex mapping is order

Example 1 Let $X$ be a Hilbert space. A mapping $F: X \rightarrow Y$ is strongly $\|\cdot\|^{2}-k_{0}$-paraconvex with constant $C \geq 0$ on a convex set $A$ if and only if the mapping $F+C\|\cdot\|^{2} k_{0}$ is $K$-convex on $A$. Indeed, let $x_{1}, x_{2} \in X$. Since

$$
\left.\lambda\left\|x_{1}\right\|^{2}+(1-\lambda)\left\|x_{2}\right\|^{2}-\| \lambda x_{1}+(1-\lambda) x_{2}\right)\left\|^{2}=\lambda(1-\lambda)\right\| x_{1}-x_{2} \|^{2}
$$

and

$$
F\left(\lambda x_{1}+(1-\lambda) x_{2}\right) \leq_{K} \lambda F\left(x_{1}\right)+(1-\lambda) F\left(x_{2}\right)+C \lambda(1-\lambda)\left\|x_{1}-x_{2}\right\|^{2} k_{0}
$$

we have

$$
\begin{aligned}
& \left.F\left(\lambda x_{1}+(1-\lambda) x_{2}\right)+C \| \lambda x_{1}+(1-\lambda) x_{2}\right) \|^{2} k_{0} \\
& \quad \leq_{K} \lambda F\left(x_{1}\right)+(1-\lambda) F\left(x_{2}\right)+C\left(\lambda\left\|x_{1}\right\|^{2} k_{0}+(1-\lambda)\left\|x_{2}\right\|^{2}\right) k_{0} .
\end{aligned}
$$

The mapping $F(\cdot)=F(\cdot)+C\|\cdot\|^{2} k_{0}$ is clearly order d.c. Furthermore, if $K$ is well based $\left(\exists y^{*} \in Y^{*}\right.$ such that $y^{*}(k) \geq\|k\|$ for any $\left.k \in K\right)$, then $F$ is d.c.

For d.c. mappings, we have the following result on the existence of directional derivative.

Theorem 1 (Proposition 3.1 of [13]) Let $X$ be a normed linear space and let $Y$ be a Banach space. Let $G \subset X$ be an open convex set and let $F: G \rightarrow Y$ be a d.c. mapping. Then, the directional derivative $F^{\prime}\left(x_{0}, h\right)$ exists whenever $x_{0} \in G$ and $h \in X$.

Let us observe that if the function $\alpha(\cdot)$ is not convex, then we cannot expect a strongly $\alpha(\cdot)-k_{0}$ paraconvex mapping $F$ to be d.c.

\section{Monotonicity of Difference Quotients}

Let $X$ be a normed space. Let $Y$ be a topological vector space and let $K \subset Y$ be a closed convex pointed cone.

For $K$-convex mappings, the difference quotient is nondecreasing in the sense that

$$
\phi\left(t_{1}\right)-\phi\left(t_{2}\right):=\frac{F\left(x_{0}+t_{1} h\right)-F\left(x_{0}\right)}{t_{1}}-\frac{F\left(x_{0}+t_{2} h\right)-F\left(x_{0}\right)}{t_{2}} \in K \quad \text { for } t_{1} \geq t_{2} .
$$

For strongly $\alpha(\cdot)-K$ paraconvex and strongly $\alpha(\cdot)-k_{0}$ paraconvex mappings, the difference quotient may not be nondecreasing. 
Example 2 Let $Y=\mathbb{R}, K=\mathbb{R}_{+}, \alpha(x)=x^{2}$ and let $F(x)=-x^{2}$. The mapping $F$ is strongly $\alpha(\cdot)-K$-paraconvex. Observe that for any $x_{1}, x_{2} \in \mathbb{R}$, we have $t\left(x_{1}^{2}+x_{2}^{2}\right)-$ $2 t\left(x_{1} x_{2}\right) \leq 0$ if and only if $t \leq 0$. Hence, for $t=-\lambda^{2}+\lambda-1 \leq 0$, we have

$$
\begin{gathered}
\left(-\lambda^{2}+\lambda-1\right)\left(x_{1}^{2}+x_{2}^{2}\right)-2 x_{1} x_{2}\left(-\lambda^{2}+\lambda-1\right) \leq 0, \\
x_{1}^{2}\left(-\lambda^{2}+\lambda-1\right)+x_{1} x_{2}(-2 \lambda(1-\lambda)+2)+x_{2}^{2}\left(-(1-\lambda)^{2}+1-\lambda-1\right) \leq 0, \\
-\left(\lambda x_{1}+(1-\lambda) x_{2}\right)^{2} \leq-\lambda x_{1}^{2}-(1-\lambda) x_{2}^{2}+\left(x_{1}-x_{2}\right)^{2}, \\
F\left(\lambda x_{1}+(1-\lambda) x_{2}\right) \leq \lambda F\left(x_{1}\right)+(1-\lambda) F\left(x_{2}\right)+\left(x_{1}-x_{2}\right)^{2} .
\end{gathered}
$$

The last inequality and Proposition 2.1 from [5] give us paraconvexity of the mapping $F$.

Let $x_{0}=0, h=1$. The difference quotient $\phi(t)=\frac{F\left(x_{0}+t h\right)-F\left(x_{0}\right)}{t}$ is decreasing. Indeed, for $t_{1} \leq t_{2}$, we have $\phi\left(t_{1}\right)=-t_{1}$ and $\phi\left(t_{2}\right)=-t_{2}$.

The following two propositions are basic tools for the proof of the main result in the next section. In the proposition below, we investigate the monotonicity properties of the $\alpha(\cdot)$-difference quotients for strongly $\alpha(\cdot)-k$ paraconvex mappings.

Proposition 1 Let $X$ be a normed space and let $Y$ be a vector space and ordered by $a$ convex pointed cone $K$. Let $F: X \rightarrow Y$ be strongly $\alpha(\cdot)-k_{0}$ paraconvex on a convex set $A \subset X$ with constant $C \geq 0, k_{0} \in K \backslash\{0\}$. For any $x_{0} \in A$ and any $h \in X,\|h\|=1$ such that $x_{0}+$ th $\in$ A for all t sufficiently small, the $\alpha(\cdot)$-difference quotient mapping $\phi: \mathbb{R} \rightarrow Y$ defined as

$$
\phi(t):=\frac{F\left(x_{0}+t h\right)-F\left(x_{0}+t_{0} h\right)}{t-t_{0}}+C \frac{\alpha\left(t-t_{0}\right)}{t-t_{0}} k_{0} \quad \text { for } t_{0}<t,
$$

where $t_{0} \in \mathbb{R}$ is $\alpha(\cdot)$-nondecreasing in the sense that

$$
\phi(t)-\phi\left(t_{1}\right)+C \frac{\alpha\left(t_{1}-t_{0}\right)}{t_{1}-t_{0}} k_{0} \in K \quad \text { for } t_{0}<t_{1}<t .
$$

Proof Take any $t_{0}<t_{1}<t$. We have $0<\lambda:=\frac{t_{1}-t_{0}}{t-t_{0}}<1$ and

$$
x_{0}+t_{1} h=\lambda\left(x_{0}+t h\right)+(1-\lambda)\left(x_{0}+t_{0} h\right) .
$$

Let $k_{0} \in K \backslash\{0\}$. Since $F$ is strongly $\alpha(\cdot)$ - $k_{0}$ paraconvex with constant $C \geq 0$, we have

$$
\begin{aligned}
F\left(x_{0}+t_{1} h\right) \leq_{K} & \lambda F\left(x_{0}+t h\right)+(1-\lambda) F\left(x_{0}+t_{0} h\right) \\
& +C \min \{\lambda, 1-\lambda\} \alpha\left(t-t_{0}\right) k_{0} .
\end{aligned}
$$

Hence,

$$
\begin{aligned}
0 \leq_{K} \quad & \lambda\left[F\left(x_{0}+t h\right)-F\left(x_{0}+t_{0} h\right)\right]-\left[F\left(x_{0}+t_{1} h\right)-F\left(x_{0}+t_{0} h\right)\right] \\
& +C \min \{\lambda, 1-\lambda\} \alpha\left(t-t_{0}\right) k_{0},
\end{aligned}
$$

i.e.,

$$
\begin{aligned}
& {\left[\frac{F\left(x_{0}+t h\right)-F\left(x_{0}+t_{0} h\right)}{t-t_{0}}\right]-\left[\frac{F\left(x_{0}+t_{1} h\right)-F\left(x_{0}+t_{0} h\right)}{t_{1}-t_{0}}\right]} \\
& +C \min \{\lambda, 1-\lambda\} \frac{\alpha\left(t-t_{0}\right)}{t_{1}-t_{0}} k_{0} \in K .
\end{aligned}
$$

We have 
(i) If $\lambda \leq 1-\lambda$, i.e., $t_{1}-t_{0} \leq t-t_{0}$, then

$$
\min \{\lambda, 1-\lambda\} \frac{\alpha\left(t-t_{0}\right)}{t_{1}-t_{0}}=\frac{\alpha\left(t-t_{0}\right)}{t-t_{0}} .
$$

(ii) If $\lambda>1-\lambda$, i.e., $\frac{t_{1}-t_{0}}{t-t_{0}}>\frac{t-t_{1}}{t-t_{0}}$, then

$$
\min \{\lambda, 1-\lambda\} \frac{\alpha\left(t-t_{0}\right)}{t_{1}-t_{0}}=\frac{t-t_{1}}{t-t_{0}} \frac{\alpha\left(t-t_{0}\right)}{t_{1}-t_{0}}<\frac{\alpha\left(t-t_{0}\right)}{t-t_{0}} .
$$

In both cases,

$$
\begin{aligned}
& {\left[\frac{F\left(x_{0}+t h\right)-F\left(x_{0}+t_{0} h\right)}{t-t_{0}}\right]-\left[\frac{F\left(x_{0}+t_{1} h\right)-F\left(x_{0}+t_{0} h\right)}{t_{1}-t_{0}}\right]} \\
& +C \frac{\alpha\left(t-t_{0}\right)}{t-t_{0}} k_{0}-C \frac{\alpha\left(t_{1}-t_{0}\right)}{t_{1}-t_{0}} k_{0}+C \frac{\alpha\left(t_{1}-t_{0}\right)}{t_{1}-t_{0}} k_{0} \in K .
\end{aligned}
$$

If int $K \neq \emptyset$, then any strongly $\alpha(\cdot)-k_{0}$ paraconvex mapping $F$ is strongly $\alpha(\cdot)-K$ paraconvex and for any $k \in K$ the $\alpha(\cdot)$-difference quotients satisfy formula (3) with different constants $C$, and in general, one cannot find a single constant $C$ for all $0 \neq k \in K$.

In the proposition below, we investigate the boundedness of $\alpha(\cdot)$-difference quotient for strongly $\alpha(\cdot)-k$ paraconvex mappings.

Proposition 2 Let $X$ be a normed space. Let $Y$ be a topological vector space and let $Y$ be ordered by a closed convex pointed cone $K$. Let $F: X \rightarrow Y$ be strongly $\alpha(\cdot)-k_{0}$ paraconvex on a convex set $A \subset X$ with constant $C \geq 0, k_{0} \in K \backslash\{0\}$.

For any $x_{0} \in A$ and any $h \in X,\|h\|=1$ such that $x_{0}+$ th $\in$ A for all $t$ sufficiently small, the $\alpha(\cdot)$-difference quotient mapping $\phi:[0,+\infty) \rightarrow Y$,

$$
\phi(t):=\frac{F\left(x_{0}+t h\right)-F\left(x_{0}\right)}{t}+C \frac{\alpha(t)}{t} k_{0}
$$

is bounded from below in the sense that there are an element $a \in Y$ and $\delta>0$ such that

$$
\phi(t)-a \in K \quad \text { for } 0<t<\delta .
$$

Proof Let us take $t_{0}=-t, t_{1}=0$. From inclusion (3), we have

$$
\frac{F\left(x_{0}+t h\right)-F\left(x_{0}-t h\right)}{2 t}+C \frac{\alpha(2 t)}{2 t} k_{0}-\frac{F\left(x_{0}\right)-F\left(x_{0}-t h\right)}{t}-C \frac{\alpha(t)}{t} k_{0}+C \frac{\alpha(t)}{t} k_{0} \in K .
$$

Multiplying both sides by $2 t>0$, we get

$$
F\left(x_{0}+t h\right)-F\left(x_{0}-t h\right)+C \alpha(2 t) k_{0}-2 F\left(x_{0}\right)+2 F\left(x_{0}-t h\right) \in K .
$$

By simple calculations, we get

$$
\frac{F\left(x_{0}+t h\right)-F\left(x_{0}\right)}{t}+\frac{F\left(x_{0}-t h\right)-F\left(x_{0}\right)}{t}+2 C \frac{\alpha(2 t)}{2 t} k_{0} \in K .
$$

Since $\lim _{t \rightarrow 0^{+}} \frac{\alpha(t)}{t}=0$, there exists $\delta>0$ such that $2 C \frac{\alpha(2 t)}{2 t} \leq 1$ for $t \in(0, \delta)$. We have

$$
\frac{F\left(x_{0}+t h\right)-F\left(x_{0}\right)}{t}+k_{0} \geq_{K}-\frac{F\left(x_{0}-t h\right)-F\left(x_{0}\right)}{t} .
$$


Now, let us take $-1<-t<0$. We have

$$
x_{0}-t h=t \underbrace{\left(x_{0}-h\right)}_{x_{1}}+(1-t) \underbrace{x_{0}}_{x_{2}} .
$$

From the $\alpha(\cdot)-k_{0}$ paraconvexity (1) for $\lambda:=t$, we get

$$
F\left(x_{0}-t h\right) \leq_{K} t F\left(x_{0}-h\right)+(1-t) F\left(x_{0}\right)+C \min \{t, 1-t\} \alpha(1) k_{0}
$$

By simple calculation, we get

$$
-\frac{F\left(x_{0}-t h\right)-F\left(x_{0}\right)}{t}-F\left(x_{0}\right)+F\left(x_{0}-h\right)+C \frac{\min \{t, 1-t\}}{t} \alpha(1) k_{0} \in K .
$$

Since $\frac{\min \{t, 1-t\}}{t}=\frac{1-|2 t-1|}{2 t}$ and the fact that $\frac{1-|2 t-1|}{2 t} \leq 1$ is bounded, we get

$$
-\frac{F\left(x_{0}-t h\right)-F\left(x_{0}\right)}{t}-F\left(x_{0}\right)+F\left(x_{0}-h\right)+C \alpha(1) k_{0} \in K .
$$

Hence,

$$
-\frac{F\left(x_{0}-t h\right)-F\left(x_{0}\right)}{t}-F\left(x_{0}\right)+F\left(x_{0}-h\right)+C \alpha(1) k_{0} \in K .
$$

From (5), we get

$$
\frac{F\left(x_{0}+t h\right)-F\left(x_{0}\right)}{t}-b \geq_{K} 0,
$$

where $b:=F\left(x_{0}\right)-F\left(x_{0}-h\right)-(C \alpha(1)+1) k_{0}$. Finally,

$$
\phi(t)-b \geq_{K} 0 \quad \text { for } 0<t<\delta .
$$

\section{Main Result}

The proof of the main theorem is based on the following lemma.

Lemma 1 Let $Y$ be a Banach space. Let $K \subset Y$ be a closed convex normal cone. Let $\Phi: \mathbb{R}_{+} \rightarrow Y$ satisfy the following conditions

(i) $\Phi(t) \in K$ for any $t \in \mathbb{R}_{+}$,

(ii) for $0<t_{1}<t$ we have $\Phi(t)-\Phi\left(t_{1}\right)+\frac{\alpha\left(t_{1}\right)}{t_{1}} k_{0} \in K$ for some $k_{0} \in K$,

(iii) $\Phi(t)$ is weakly convergent to 0 when $t \rightarrow 0^{+}$.

Then, $\|\Phi(t)\| \rightarrow 0$ when $t \rightarrow 0^{+}$.

Proof By contradiction, suppose that $\|\Phi(t)\| \nrightarrow 0$ when $t \rightarrow 0^{+}$and (i) and (ii) are satisfied. We will obtain a contradiction with (iii). By this, there is $\varepsilon>0$ such that for all $\delta>0$ one can find $0<t<\delta$ with $\|\Phi(t)\|>\varepsilon$. In particular, for $\delta_{n}=\frac{1}{n}$, there exist $t_{n} \in\left(0, \frac{1}{n}\right)$, $n \in \mathbb{N}$, such that

$$
\left\|\Phi\left(t_{n}\right)\right\|>\varepsilon .
$$


Let $x \in A:=\operatorname{co}\left(\Phi\left(t_{n}\right), n \in \mathbb{N}\right)$. There are positive numbers $\lambda_{1}, \lambda_{2}, \ldots, \lambda_{m}$ and $t_{1}, t_{2} \ldots, t_{m}$ such that $x=\sum_{i=1}^{m} \lambda_{i} \Phi\left(t_{i}\right)$, where $\sum_{i=1}^{m} \lambda_{i}=1$. There exists $N \in \mathbb{N}$ such that for all $n>N$, we have

$$
\begin{gathered}
\Phi\left(t_{1}\right)-\Phi\left(t_{n}\right)+\frac{\alpha\left(t_{n}\right)}{t_{n}} k_{0} \in K, \\
\Phi\left(t_{2}\right)-\Phi\left(t_{n}\right)+\frac{\alpha\left(t_{n}\right)}{t_{n}} k_{0} \in K, \\
\vdots \\
\Phi\left(t_{m}\right)-\Phi\left(t_{n}\right)+\frac{\alpha\left(t_{n}\right)}{t_{n}} k_{0} \in K .
\end{gathered}
$$

We get

$$
x-\Phi\left(t_{n}\right)+\frac{\alpha\left(t_{n}\right)}{t_{n}} k_{0} \in K \quad \text { for all } n>N .
$$

From the fact that $\Phi\left(t_{n}\right) \in K$ and $K$ is normal, there is some $c>0$ such that $\left\|\Phi\left(t_{n}\right)\right\| \leq$ $c\left\|x+\frac{\alpha\left(t_{n}\right)}{t_{n}} k_{0}\right\|$. By (6), we obtain $\left\|x+\frac{\alpha\left(t_{n}\right)}{t_{n}} k_{0}\right\|>\beta:=\frac{\varepsilon}{c}$ for all $x \in A$ and $n>N$.

We show that

$$
\mathbb{B}_{\beta / 2} \cap\left(A+k_{0}[0, s]\right)=\varnothing
$$

for $s>0$ satisfying $\frac{\alpha\left(t_{n}\right)}{t_{n}} \leq s$. To see this, take any $\ell \in(0, s]$, where $\mathbb{B}_{r}:=\{y \in Y:\|y\| \leq$ $r$. Since $\lim _{n \rightarrow+\infty} \frac{\alpha\left(t_{n}\right)}{t_{n}}=0$, there exists $n \in \mathbb{N}$ such that

$$
0 \leq_{K} x+\frac{\alpha\left(t_{n}\right)}{t_{n}} k_{0} \leq_{K} x+\ell k_{0} .
$$

By (6) and the normality of $K$,

$$
\beta / 2<\left\|x+\frac{\alpha\left(t_{n}\right)}{t_{n}} k_{0}\right\| \leq\left\|x+\ell k_{0}\right\| .
$$

From the Hahn-Banach theorem applied to $\mathbb{B}_{\beta / 2}$ and $\left(A+k_{0}[0, s]\right)$, there is a linear functional $y^{*} \in Y^{*}$ and $r>0$ such that

$$
y^{*}\left(x+\ell k_{0}\right)>r \quad \text { for all } x+\ell k_{0} \in A+k_{0}[0, s] .
$$

In particular, $y^{*}\left(\Phi\left(t_{n}\right)+\frac{\alpha\left(t_{n}\right)}{t_{n}} k_{0}\right)>r>0$, which contradicts (iii).

We are in a position to prove our main result.

Theorem 2 Let $X$ be a normed space. Let $Y$ be a weakly sequentially complete Banach space ordered by a closed convex normal cone $K$. Let $F: X \rightarrow Y$ be strongly $\alpha(\cdot)-k_{0}$ paraconvex on a convex set $A \subset X$ with constant $C \geq 0, k_{0} \in K \backslash\{0\}$. Then, the directional derivative

$$
F^{\prime}\left(x_{0} ; h\right):=\lim _{t \rightarrow 0^{+}} \frac{F\left(x_{0}+t h\right)-F\left(x_{0}\right)}{t}
$$

of $F$ at $x_{0}$ exists for any $x_{0} \in A$ and any direction $0 \neq h \in X,\|h\|=1$ such that $x_{0}+$ th $\in A$ for all $t$ sufficiently small.

Proof Let $x_{0} \in A$ and let $0 \neq h \in X,\|h\|=1$ be such that $x_{0}+t h \in A$ for all $t$ sufficiently small. Let $t_{n} \downarrow 0$. For $t_{0}=0$, the $\alpha(\cdot)$-difference quotient by (2) takes the form

$$
\phi\left(t_{n}\right)=\frac{F\left(x_{0}+t_{n} h\right)-F\left(x_{0}\right)}{t_{n}}+C \frac{\alpha\left(t_{n}\right)}{t_{n}} k_{0} .
$$


Let $y^{*} \in K^{*}$. By (4), the sequence $a_{n}:=y^{*}\left(\phi\left(t_{n}\right)\right), n \in \mathbb{N}$ is bounded from below, i.e.,

$$
a_{n} \geq a:=y^{*}(b) \text { for all } n \text { sufficiently large and } b \in Y \text {. }
$$

Let us take $\varepsilon>0$. There is $N$ such that

$$
a_{N}<\underline{a}+\frac{\varepsilon}{2},
$$

where $\underline{a}:=\inf \left\{a_{n}: n \in \mathbb{N}\right\}$. Since $\left\{t_{n}\right\}$ is decreasing, from (3), we get

$$
a_{N}-a_{n}+C \frac{\alpha\left(t_{n}\right)}{t_{n}} y^{*}\left(k_{0}\right) \geq 0 \text { for } n>N .
$$

Let $b_{n}:=C \frac{\alpha\left(t_{n}\right)}{t_{n}} y^{*}\left(k_{0}\right)$. Since $b_{n} \rightarrow 0$ there is $N_{1}$ such that $b_{n} \leq \frac{\varepsilon}{2}$ for $n>N_{1}$.

From (7) and (8), we get

$$
\underline{a}-\varepsilon<\underline{a} \leq a_{n} \leq a_{N}+b_{n} \leq \underline{a}+\frac{\varepsilon}{2}+b_{n} \leq \underline{a}+\varepsilon \quad \text { for } n>\max \left\{N, N_{1}\right\} .
$$

Hence, the sequence $\left\{a_{n}\right\}$ is convergent and consequently every sequence $\left\{y^{*}\left(\phi\left(t_{n}\right)\right)\right\}$ is Cauchy for $y^{*} \in K^{*}$.

Let us take any $h^{*} \in Y^{*}$. We show that the sequence $\left\{h^{*}\left(\phi\left(t_{n}\right)\right)\right\}$ is Cauchy. From the fact that $K$ is normal, we have $Y^{*}=K^{*}-K^{*}$ and $h^{*}=g^{*}-q^{*}$ with $g^{*}, q^{*} \in K^{*}$. Since $\left\{g^{*}\left(\phi\left(t_{n}\right)\right)\right\}$ and $\left\{q^{*}\left(\phi\left(t_{n}\right)\right)\right\}$ are Cauchy sequences, there exist $N_{1}, N_{2}$ such that for $n, m>\bar{N}:=\max \left(N_{1}, N_{2}\right)$, we have

$$
\left|g^{*}\left(\phi\left(t_{n}\right)\right)-g^{*}\left(\phi\left(t_{m}\right)\right)\right| \leq \frac{\varepsilon}{2} \quad \text { and } \quad\left|q^{*}\left(\phi\left(t_{n}\right)\right)-q^{*}\left(\phi\left(t_{m}\right)\right)\right| \leq \frac{\varepsilon}{2} .
$$

For $n>\bar{N}$, we have

$$
\begin{aligned}
\left|h^{*}\left(\phi\left(t_{n}\right)\right)-h^{*}\left(\phi\left(t_{m}\right)\right)\right| & =\left|g^{*}\left(\phi\left(t_{n}\right)\right)-q^{*}\left(\phi\left(t_{n}\right)\right)-g^{*}\left(\phi\left(t_{m}\right)\right)+q^{*}\left(\phi\left(t_{m}\right)\right)\right| \\
& \leq \frac{\varepsilon}{2}+\frac{\varepsilon}{2}=\varepsilon .
\end{aligned}
$$

We show that $\phi(t)$ weakly converges when $t \rightarrow 0^{+}$, i.e., there is an $y_{0} \in Y$ such that for arbitrary $t_{n} \downarrow 0$, we have

$$
\lim _{n \rightarrow \infty} y^{*}\left(\phi\left(t_{n}\right)\right)=y^{*}\left(y_{0}\right) \quad \text { for any } y^{*} \in Y^{*}
$$

which is equivalent to

$$
\phi(t) \rightarrow y_{0} \quad \text { when } t \rightarrow 0^{+} .
$$

Since $Y$ is weakly sequentially complete, we need only to show that $y_{0}$ is the same for all sequences $\left\{t_{n}\right\}, t_{n} \downarrow 0$. On the contrary, suppose that there are two different weak limits $y_{0}^{1}, y_{0}^{2}$ corresponding to sequences $t_{n}^{1}$ and $t_{n}^{2}$, respectively.

We can subtract subsequences $\left\{\bar{t}_{n}^{2}\right\} \subset\left\{t_{n}^{2}\right\}$ and $\left\{\bar{t}_{n}^{1}\right\} \subset\left\{t_{n}^{1}\right\}$ such that $\bar{t}_{n}^{2} \leq t_{n}^{1} \leq \bar{t}_{n}^{1}$. Correspondingly,

$$
y^{*}\left(\phi\left(\bar{t}_{n}^{2}\right)\right) \leq y^{*}\left(\phi\left(t_{n}^{1}\right)\right) \leq y^{*}\left(\phi\left(\bar{t}_{n}^{1}\right)\right)
$$

which proves that it must be $y_{0}^{1}=y_{0}^{2}$.

Now, we show that the mapping $\Phi(t):=\phi(t)-y_{0}$ satisfies all the assumptions of Lemma 1. From (3) and (9), it is enough to show that $\Phi(t) \in K$ for all $t \geq 0$.

By contradiction, let us assume that there is some $\bar{t}>0$ such that $\Phi(\bar{t}) \notin K$. There exists $y^{*} \in K^{*}$ such that

$$
y^{*}(\Phi(\bar{t}))=y^{*}\left(\phi(\bar{t})-y_{0}\right)<0 .
$$

From inclusion (3) in Proposition 1, we have

$$
\phi(\bar{t})-y_{0}-\phi(t)+y_{0}+C \frac{\alpha(t)}{t} k_{0} \in K \quad \text { for all } t \in(0, \bar{t}) .
$$


In particular

$$
y^{*}\left(\phi(\bar{t})-y_{0}\right) \geq y^{*}\left(\phi(t)-y_{0}-C \frac{\alpha(t)}{t} k_{0}\right) \quad \text { for all } t \in(0, \bar{t}) .
$$

And by (10), we get

$$
0>y^{*}\left(\phi(\bar{t})-y_{0}\right) \geq y^{*}\left(\phi(t)-y_{0}-C \frac{\alpha(t)}{t} k_{0}\right) \quad \text { for all } t \in(0, \bar{t}) .
$$

Then, by letting $t \rightarrow 0^{+}$, we get contradiction with (9). By Lemma $1, \Phi(t)$ tends to 0 when $t \rightarrow 0^{+}$. Since $\lim _{t \rightarrow 0^{+}} \frac{\alpha(t)}{t}=0$, we get

$$
\lim _{t \rightarrow 0^{+}} \frac{F\left(x_{0}+t h\right)-F\left(x_{0}\right)}{t}=y_{0}
$$

which completes the proof.

Remark 1 For $K$-convex mappings $F$, i.e., strongly $\alpha(\cdot)$ - $K$ paracanovex mappings with constant $C=0$ Theorem 2 can be found in [12].

Open Access This article is distributed under the terms of the Creative Commons Attribution 4.0 International License (http://creativecommons.org/licenses/by/4.0/), which permits unrestricted use, distribution, and reproduction in any medium, provided you give appropriate credit to the original author(s) and the source, provide a link to the Creative Commons license, and indicate if changes were made.

\section{References}

1. Cannarsa, P., Sinestrari, C.: Semiconcave Functions, Hamilton-Jacobi Equation and Optimal Control. Progress in Nonlinear Differential Equations and Their Applications, vol. 58. Birkhäuser, Boston (2004)

2. Daniilidis, A., Georgiev, P.: Approximate convexity and submonotonicity. J. Math. Anal. Appl. 291, 292-301 (2004)

3. Huang, H., Li, R.: Global error bounds for $\gamma$-paraconvex multifunctions. Set-Valued Var. Anal. 19, 487-504 (2011)

4. Huang, H.: Coderivative conditions for error bounds of $\gamma$-paraconvex multifunctions. Set-Valued Var. Anal. 20, 567-579 (2012)

5. Jourani, A.: Subdifferentiability and subdifferential monotonicity of $\gamma$-paraconvex functions. Control Cybern. 25, 721-737 (1996)

6. Ngai, H.V., Luc, D.T., Théra, M.: Approximate convex functions. J. Nonlinear Convex Anal. 1, 155-176 (2000)

7. Rolewicz, S.: On paraconvex multifunctions. Oper. Res. Verf. (Methods Oper. Res.) 31, 540-546 (1979)

8. Rolewicz, S.: On $\gamma$-paraconvex multifunctions. Math. Japonica 24B, 293-300 (1979)

9. Rolewicz, S.: On $\alpha(\cdot)$-paraconvex and strongly $\alpha(\cdot)$-paraconvex functions. Control Cybern. 29, 367-377 (2000)

10. Rolewicz, S.: Differentiability of strongly paraconvex vector-valued functions. Funct. Approx. Comment. Math. 44, 273-277 (2011)

11. Tabor, J., Tabor, J.: Paraconvex, but not strongly, Takagi functions. Control Cybern. 41, 545-559 (2012)

12. Valadier, M.: Sous-Différentiabilité de fonctions convexes à valeurs dans un espace vectoriel ordonné. Math. Scand. 30, 65-74 (1972)

13. Veselý, L., Zajíček, L.: Delta-Convex Mappings between Banach Spaces. Dissertationnes Mathematicae, vol. 289 (1989)

14. Veselý, L., Zajíček, L.: On D.C. mappings and differences of convex operators. Acta Univ. Carol. Math. Phys. 42, 89-97 (2001)

15. Veselý, L., Zajíček, L.: On connections between delta-convex mappings and convex operators. Proc. Edinb. Math. Soc. 49, 739-751 (2006)

16. Veselý, L., Zajíček, L.: On composition of d.c. functions and mappings. J. Convex Anal. 16, 423-439 (2009) 